\title{
A Dual Fuzzy Entropy Model for QoS Routing in MANET
}

\author{
Uday.S.B \\ P.D.A College of Engineering \\ Gulbarga \\ Karnataka,India
}

\author{
K. Karibasappa \\ Vice Principal \\ Dayanand Sagar Engineering \\ College Bengalooru,Karnataka,India
}

\author{
V.D. Mytri \\ Principal \\ G.N.D College of Engineering \\ Bidar,Karnataka
}

\begin{abstract}
Congestion leads to bandwidth degradation and, packet losses thereby causing retransmissions and higher energy drain rate by the nodes in Mobile Ad hoc Networks(MANET). So providing Quality of Service $(\mathrm{QoS})$ and reducing congestion levels are some of the major issues to be addressed in these networks. However, use of appropriate congestion control measures is complicated due to the interdependence of various parameters that cause congestion. Applying cost based metrics for attaining QoS is prevalently used in MANET's but they are inefficient because of the uncertainities involved in the channel characteristics. Hence routing solutions based on the principles of entropy and fuzzy logic would be appropriate for MANET's. We are proposing a novel method based on entropy and fuzzy logic (Dual-Fuzzy-Entropy-AODV) to assess the quality of a path discovered by a routing protocol. Simulation studies and evaluation of our proposed mechanism shows improvement in the performance results.
\end{abstract}

\section{General Terms}

Mobile ad hoc networks, QoS Routing Algorithms.

\section{Keywords}

Entropy, Fuzzy based Routing.

\section{INTRODUCTION}

A mobile adhoc network is a wireless network environment with autonomous collection of nodes and no fixed infrastructure. The communication between the nodes is carried out through direct wireless link or with the help of other intermediate nodes between a source and destination and without any fixed base station as in the case of most infrastructure based wireless network. The performance and quality of the communication therefore depends upon the link quality of independent links existing between different mobile nodes. Link quality is a probabilistic random function that depends upon many other constraints like energy, power, mobility, packet rate, interference, and so on. Many of the studies have considered each to be independent metric and hence solutions for MANET are based on optimization of one or more such parameters. In reality ,most of these parameters are interrelated with each other. For example ,as the energy of the node decreases, transmission power is also decreased which results in more packet losses, which in turn requires packet retransmission and route or link maintenance causing an increase in delay. The increase in delay results in congestion in the network which affects over all throughput of the network. But there exists no direct relationship amongst the parameters and hence it is difficult to assume the affect of a particular parameter on the performance without correlating to other parameters. Over any observation window, each of these independent parameters may have infinite set of values. Modeling these values into a model, suitable for taking up transmission or forwarding decisions is a difficult task as the probability of the current set or state of values to be retained for the next observation depends upon many other parameters in the network. Hence it is difficult to extract the exact quantitative information measure for a variable or parameter over an interval of time. A qualitative information measure is better equivalence for the quantitive measure. For example, if the energy of group of nodes in between a source and destination is low, packet delivery ratio can be expected to be lower than expected. On the contrary if the relative mobility of the nodes are high, then the packet delivery ratio may be much lower than expected, and delay may be more. In summary the affect of various parameters can be measured as a constraint of quality of transmission rather than their distinct values over the time or any instance of time.

In the following section we deal with review of literature followed by motivation and proposed system design in section 3 and 4 simultaneously. Simulation and discussion of results is taken up in section 5 followed by conclusion in section 6

\section{REVIEW OF LITERATURE}

Quantifying the uncertainity involved in routing for computer communication networks using entropy has been introduced in [1]. This study reveals the superiority of entropy constrained routing algorithm over neural optimization approaches. [2] provides an analysis and critical review of two metrics, relative motion entropy and energy entropy. They conclude by their study that the relative motion entropy metric lacks the features to be useful for characterizing MANET node motion predictability, while the maximum energy entropy criterion leads to effective energy use by clusterheads communicating with cluster members. However, the use of information entropy analogues is unnecessary if the metrics are used individually. Under those conditions, the parameters defined in the entropy framework can be evaluated directly. [3] Proposes a reactive route handoff method which can increase the overhead from frequent route discoveries. This paper proposes a novel Entropy-based Long-life Multipath Routing algorithm in MANET (ELMR). The key idea of ELMR algorithm is to construct the new metric, entropy and select the stable multipath with the help of entropy metric to reduce the number of route reconstructions, so as to provide QoS guarantee in MANET. It is typically proposed in order to increase the reliability of data transmission or to provide load balancing. The simulation results show that the entropy based approach and parameters provide an accurate and efficient method of estimating and evaluating the route stability in dynamic MANETs.

[4] presents entropy constrained solution for power-conserved routing in Mobile Ad - Hoc networks. Experimental evaluation of this study reveals that entropy based routing solution can potentially increase the lifetime of wirless networks, when compared to the conventional routing approach using dijkstra's 
algorithm. [5] proposes an entropy based modeling framework for supporting route stability in mobile ad hoc networks.Results show that entropy based approach provide an accurate and efficient method of estimating and evaluating the route stability.

[6] proposes a new metric for reducing the probability of link breakage and route reparation using connection entropy for multiRate ad hoc networks. In [7] mobile ad hoc networks (MANETs), the provision of quality of service (QoS) guarantees is much more challenging than in wireline networks, mainly due to node mobility, multihop communications, contention for channel access, and a lack of central coordination. This paper introduces an Entropy-based Fuzzy controllers QoS Routing algorithm in MANET (EFQRM). EFQRM algorithm constructs the new metricentropy and fuzzy controllers with the help of entropy metric to reduce the number of route reconstruction so as to provide QoS guarantee in the ad hoc network. The simulation results show that the proposed approach and parameters provide an accurate and efficient method of estimating and evaluating the route stability in dynamic MANETs.

[8] A mobility metric is proposed for the unbiased comparison of networks. This is again an entropy measure based on the uncertainty of change in the topology of the network, and is referred to as topological uncertainty. Topological uncertainty determines the minimum overhead required by the network to correctly identify the topology and hence, provide node connectivity. Topological uncertainty is used to derive fundamental bounds on the maximum bit rate available within a mobile ad-hoc networking environment. This work demonstrates the potential of entropy measures to describe the complexities of node connectivity within wireless networks.

[9] applies fuzzy control to dynamic allocation of network bandwidth based on message precedence-weighted performance of MANET's that carry multiple-precedence traffic .[10] suggests the use of information theoretic metric to select cluster heads. Three metrics mobility, energy and degree are used to find the total entropy.

\section{MOTIVATION}

QOS and Congestion control are two major issues in MANET and are affected by several parameters and characteristics of the Mobile nodes and the network itself. Interdependency of the various factors fuzzifies the performance which leads to challenges in designing an optimum solution. Conventional Congestion control mechanisms like scheduling and load balancing inherently depends upon the route quality itself. Hence the core challenge in this direction can be stated as finding the routes most well suited for optimum transmission performance. Cost consideration for routing must be such that, it resolves the fuzziness amongst the parameter dependencies. Though several techniques are proposed in this direction which include both QOS and Fuzzy routing, there exists a technical drawback in these techniques. Pure QOS routing is instantaneous. It considers the performance of a node only at the time of route selection and do not take into account the history of the nodes. Entropy based solutions finds the best path depending upon the probability of performance generated from the history of the nodes but again fails to resolve the fuzziness of the parameters. Fuzzy Entropy routing fuzzifies the entropy values but fails to incorporate the result of past success of the decision in the current context. Therefore in this paper we propose a Fuzzy-Entropy-Fuzzy based routing that not only inter-relate the parameters and their dependencies, but at the same time incorporate the performance of thus obtained routes. Results shows significant performance improvement over both Entropy based routing as well as pure Fuzzy based Routing.

\section{PROPOSED SYSTEM}

The fact that, multiple parameters affect a particular transmission state at various stages of transmission is already elaborated, the transmission quality may be defined as a set of known qualitative values like good, average, better, very good, poor, unacceptable which are produced by pure random state of observed parameters like energy, power and so on. The quality of a transmission is further considered to be affected by quality or range of the observed parameters. For example a "low" energy intermediate node contributes to lesser packet delivery ratio. Thus MANET can be considered as a system where the range of values for certain parameters results in certain quality of transmission. The system can be viewed as in figure 1 .

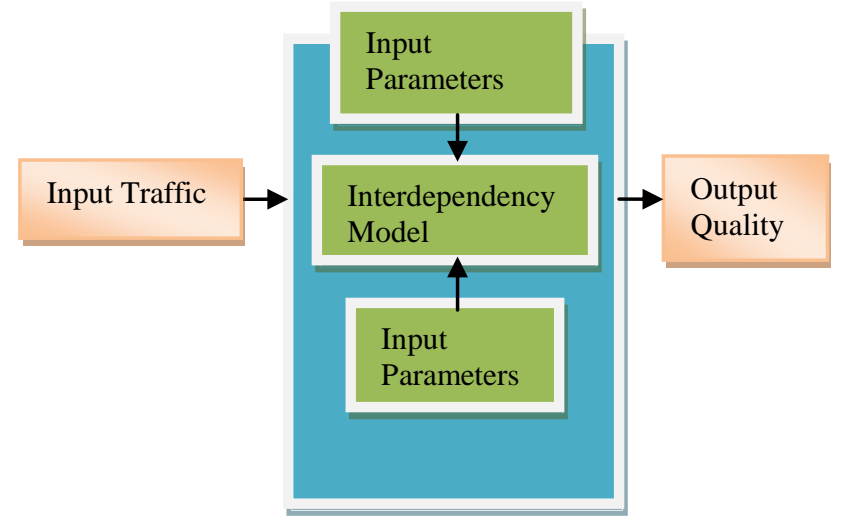

Figure 1: An abstract Model of Quality of Transmission

From figure 1 it is clear that output quality of the system is dependent on the inter dependability model and how different parameters contribute to this model. Therefore the model can be considered as a fuzzy model with definite number of sets in the output stage.

Now each of these states contains structured information and they are never pure random over the time. For example ,if in the current observation the transmission quality is very high, it can never become unacceptable in the very next observation. Such changes are gradual while moving from one state to another. An average transmission quality may get improved to be better or may further get degraded to poor but may not be unacceptable. Therefore it may be assumed that next observed output quality will be directly dependent on the current state of values.

But this assumption does not hold good in MANET all the time. For example two nodes are at the absolute boundary of accepted radio range and each node has enough energy and other parameters resulting in good quality of service. But in the next instance, they move further thus reducing the quality of the transmission to unacceptable limits. Thus the quality of transmission and the state can best be measured with a probability based on the past observations. For example, the probability of next output state being "good" is high if the past observations are also high and the next being moderate or low for the same condition. Therefore the quality of transmission with current conditions can be predicted for the next observation if the mutual information amongst the states is known.

The model of extracting the mutual information amongst the states which are assumed to be random variable is known as an 
entropy model and from Shannon entropy formulation [11] can be given by the equation 1 .

The entropy $\mathrm{H}$ of a discrete random variable $X$ with possible values $\left\{x_{1}, \ldots, x_{n}\right\}$ is

$$
H(X)=E(I(X))
$$

Here $\mathrm{X}$ can be considered as quality of transmission which is defined as set of states \{ "unacceptable", "poor", "moderate", "Good", "better", "Very good"\}.Here E is the expected value, and $I$ is the information content of $X . I(X)$ is a random variable and if $p$ denotes the probability mass function of $X$,then the entropy can explicitly be written as

$$
H(X)=\sum_{i=1}^{n} p\left(x_{i}\right) I\left(x_{i}\right)=-\sum_{i=1}^{n} p\left(x_{i}\right) \log _{{ }_{10}} p\left(x_{i}\right)
$$

From (2) it is quite clear that the smaller the values of probability of occurrence of the states, larger will be entropy values and larger the probability of occurrence of states, smaller will be entropy. Therefore large entropy represents less information content for a set of observations. In other words, if we consider two paths with different entropies, the path with lowest entropy signifies least randomness and more predictable path condition. This is the basis of assumption of our work.

Figure 2 Represents the Entropy Model for the proposed work. Now consider that in a path "poor" transmission condition is persisting and without any other type of states being observed. Now the entropy of the path will be 0 because "poor" state is occurring with probability 1 . Even though randomness in the path is minimum, the path quality can not be considered to be best. In summary, we can interpret that a transmission path can be considered as best if it satisfies the QOS parameters and also the entropy value of the path is low. Because the entropy is merely a probability of information content being consistent, it is highly impossible to set a threshold for entropy and therefore classification of entropy into small or high is quite difficult. In general, values nearer to 0 suggest low entropy and values greater than 1 , are considered as higher entropy. Now suppose over the defined set of states only "good" and "very good" states are observed, then probability of the rest of the states will be zero and the entropy value will essentially be infinity. But still the path condition is better. Hence this is very much true, that merely thresholding an entropy information never reveals the quality of the path.

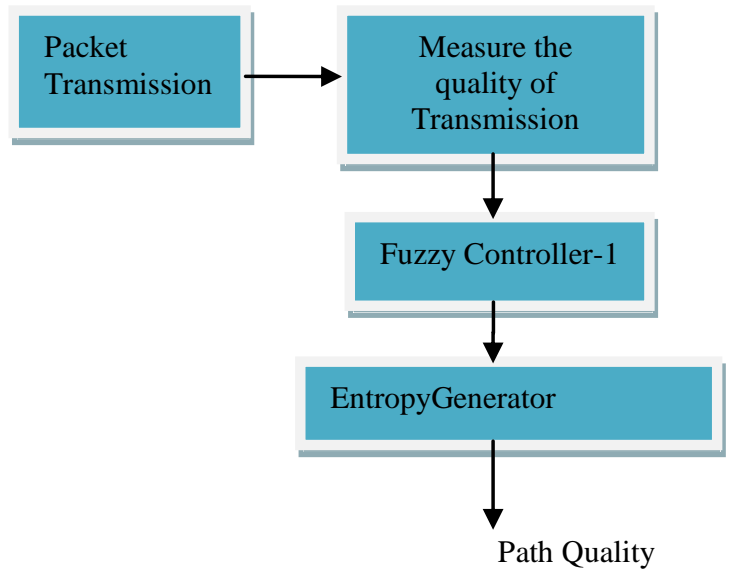

Figure 2: Model guided by Fuzzy controller and Entropy for evaluting path quality.
Hence, we define fuzzy controller for assessing the level of entropy based on the maximum observed state.This approach is presented in Figure 2, which is further modified for better approximation of path quality and is shown in Figure 3.

\subsection{Design of Fuzzy System}

A fuzzy state is one where, value of a parameter can have more than one state. For example ,if we measure the delay that a packet has suffered, then normally that delay can be considered as low or high based on a threshold value.

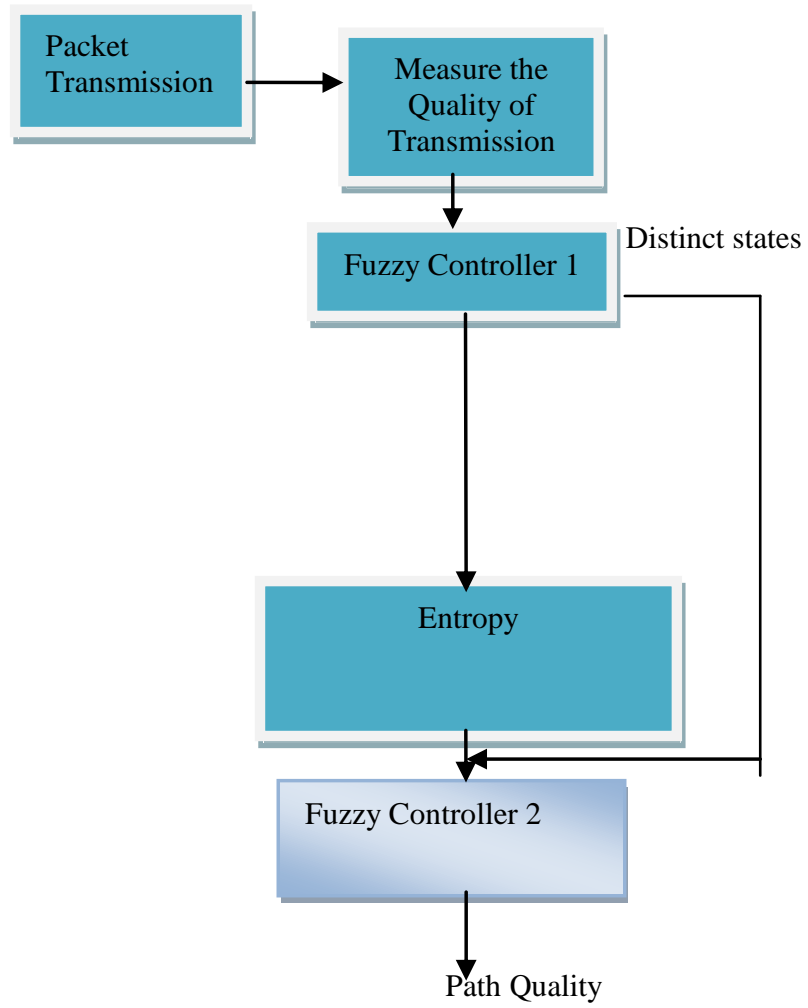

Figure 3. Block Diagram of the overall model for estimating the path Quality.

Such a state is known as binary state. But if we have different benchmarks for estimating the quality of transmission marked with different overlapping thresholds then delay may be considered as "low" "very low", "medium" high so on. Such a state is now known as fuzzy state. Now consider that the transmission quality is a pure measure of delay and is inverse function of it. Then if delay is low , transmission quality is "high" and so on. But if there exists one more parameter, say reception power then the transmission quality needs to correlate to this variable too for correct quality assessment But consider that the parameter reception power has only three states "good", "average" and "Bad" and that the transmission quality is a measure of both the parameters i.e. delay and power, then it becomes very difficult to estimate what would be transmission quality interpretation for different permutations of delay and power. The logic model that solves this problem is known as fuzzy logic[12][13]. Following steps enumerates the process of fuzzification.

1.The system must first extract the states from values called Fuzzification.

2.A table is built called a rule set which elaborates the possible value of output for every combination of input and the mechanism is called Fuzzy Rule Set.

3.States of each parameter are modeled to obtain an estimation of outcome or output. This is called Defuzzification. 
Interpreting the states from values which are threshold based and because fuzzy logic defines several states for a parameter, there are many ranges, each for a particular state. The interpolation of the threshold over entire range of the value of a parameter is called Membership function and it may take any trignometric form such as triangle, rectangle, trapezoidal. The most adaptive one is being the triangular membership function. Figure 4 shows the block diagram of a fuzzy logic system.

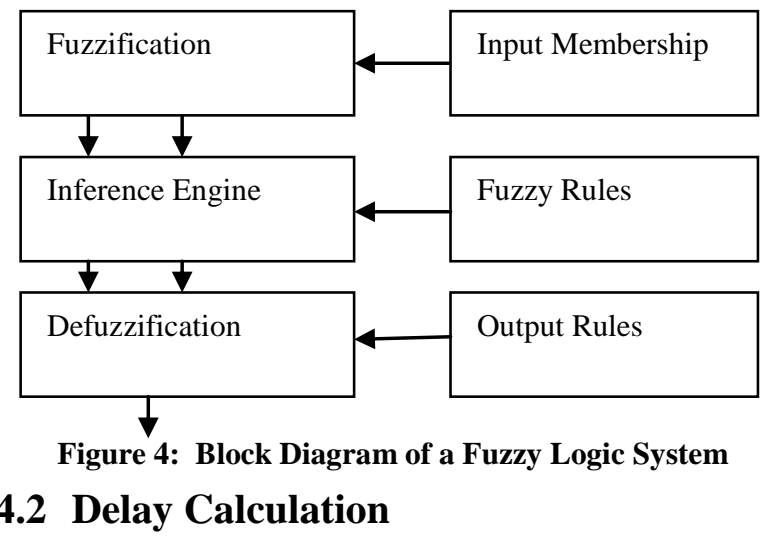

There are several techniques for calculating delay in a packet. Delays are calculated either as average end to end delay or round trip delay and so on. Delay in a transmission is caused at different layers. For example, queuing delay in MAC layer, propagation delay in physical layer, routing delay in network layer. Delay between two nodes may be calculated from the channel acquisition mechanism that is by estimating the time differential between generations of RTS and getting a CTS and so on.

Current model of estimating delay is based on probability density function of the delay values. Therefore the instantaneous values of delay are much better than the delay estimated by MAC layer at the time of channel acquisition phase. Hence we add an extra field in each packet header called START_TIME. Whenever a packet is generated, the generation time is embedded in that field. When a node receives the packet, it calculates the delay by subtracting the START_TIME from RECEIVED_TIME which is the time when the packet is received by the node. Further if this packet needs to be forwarded, the START_TIME is updated with RECEIVED_TIME and forwarded such that the next node knows the delay with respect to the last transmission.

\subsection{Signal To Noise Ratio}

SNR is a good measure of the link quality[15]. High SNR ensures higher packet delivery ratio. In a signal level measurement, calculation of SNR is typically complex. It is also calculated from the power spectrum of the received signal. But in this work, we propose a packet level estimation of SNR which can be directly derived from the bit error rate. Bit error rate (BER) of a communication system is defined as the ratio of number of error bits and total number of bits transmitted during a specific period. It is the likelihood that a single error bit will occur within received bits, independent of rate of transmission.BER can be measured by comparing the transmitted signal with the received signal and computing the error count over the total number of bits. For any given modulation, the BER is normally expressed in terms of signal to noise ratio (SNR).

It is quite difficult to form a model which can appropriately give the estimation of interference level and the power spectral density of the Gaussian noise present in the channel. As SNR and BER are closely interrelated,we use the theoretical limit table for estimating SNR from BER[14] .

Erroneous bits are calculated using parity check of the received packet. A node stores the number of packets it has received and the current measure of bit error rate. As the simulation is using 20 packets to 50 packets per second as the data transmission rate, maximum error rate that can be observed per second is $\mathrm{e}^{-7}$. Thus any value over it may be considered as infinitely low BER and infinitely high SNR Further an argument can be easily put forward about selecting SNR as the performance measurement criteria instead of BER where it is derived directly from the table. Error rate are more instantaneous values. For example if SNR is around $4 \mathrm{~dB}$, the BER rate may vary from $4 \mathrm{e}^{-2}$ to $8 \mathrm{e}^{-3}$.

Table 1. Theoretical limit table for estimating SNR from BER

\begin{tabular}{|l|l|l|l|}
\hline SNR(dB) & $\begin{array}{l}\text { BPSK\& } \\
\text { QPSK }\end{array}$ & CCK5.5(Mbps) & CCK11(11Mbps) \\
\hline 4 & $9 \mathrm{e}-2$ & $1.7 \mathrm{e}-2$ & $4 \mathrm{e}-2$ \\
\hline 5 & $9 \mathrm{e}-2$ & $3.5 \mathrm{e}-3$ & $8 \mathrm{e}-3$ \\
\hline 6 & $3 \mathrm{e}-2$ & $6.2 \mathrm{e}-4$ & $1.2 \mathrm{e}-3$ \\
\hline 7 & $4.1 \mathrm{e}-3$ & $7 \mathrm{e}-5$ & $1.2 \mathrm{e}-4$ \\
\hline 8 & $1.01 \mathrm{e}-3$ & $5 \mathrm{e}-6$ & $1.01 \mathrm{e}-5$ \\
\hline 9 & $2 \mathrm{e}-4$ & $2 \mathrm{e}-7$ & $3.9 \mathrm{e}-7$ \\
\hline 10 & $2.02 \mathrm{e}-5$ & $4 \mathrm{e}-9$ & $7 \mathrm{e}-9$ \\
\hline 11 & $1.8 \mathrm{e}-6$ & $7 \mathrm{e}-11$ & $2 \mathrm{e}-10$ \\
\hline 12 & $5.9 \mathrm{e}-8$ & $7 \mathrm{e}-11$ & $2 \mathrm{e}-10$ \\
\hline 13 & $1.1 \mathrm{e}-9$ & $7 \mathrm{e}-11$ & $2 \mathrm{e}-10$ \\
\hline
\end{tabular}

But if bit error rate is directly considered for parameter monitoring, then each of distinct BER values will have to be considered as independent state and number of states will be very high which further makes it difficult to model a fuzzy system or for that matter the entropy model.

\subsection{Methodology}

All the nodes upon receiving packets( either data or control) calculates SNR from BER and the delay from the packets. As the route request arrives at the node with a certain QoS demand, the node first defuzzifies the parameters and compares it with the required QoS. If the QoS demand is met, it generates the entropy using the output of fuzzy controller1. Next using entropy values as inputs, fuzzy-controller 2 resolves and stores the entropy values from the parameters and the fuzzy result in the RREQ packet. The destination waits to receive further RREQ's . Destination node then selects the path whose average fuzzy value matches with that of the QoS specified by the source .If the one or more paths satisfy the QoS demands then ,destination selects a path with least uncertainity or in otherwords a path with lowest entropy and generates a route reply(RREP) packet which then is sent along the reverse path. Low entropy suggests least variability in the path and ascertain good transmission quality. Therefore the path not only guarantees good quality transmission but also the same for an extended period of time is guaranteed. During the course of transmission if there are path losses due to mobility or energy drainage at the nodes, RERR or route error packets are generated and the particular path is removed from the cache. 


\section{SIMULATION}

The proposed technique (Dual-Fuzzy-Entropy-AODV) is simulated in OMNet++[16], a parallel discrete event simulator to analyze the performance of the system which is presented in the following section.

Simulation was conducted using 200 mobile nodes moving randomly at a speed of 0 to 20 metres per second in an area of $1000 \times 1000$ metres . Mobility of the nodes is simulated using Random Way Point model. IEEE 802.11b MAC is assumed. Pause time of the nodes is kept at 0 to 20 seconds. Packet size is assumed to be 512 bits. BER is kept at $1 \times 10^{-6}$.Every node is assumed to transmit with a power of 5 mwatt per packet and has an initial energy of 1000 mjoules and bandwidth is at 11 Mbps.

\subsection{Results and Discussion}

Performance parameters studied are packet delivery ratio, latency and control overhead by varying the load and the number of sessions. Efficiency of the proposed protocol is compared with that of AODV[17]. Instead of evaluating directly the proposed protocol, we have taken up a step by step evaluation. Initiailly we adopt only fuzzy decision enhancement to AODV (Fuzzy-AODV). Next entropy based decision is incorporated in to AODV which is Entropy-AODV. Then we combine entropy based decision making along with fuzzy (Fuzzy-Entropy-AODV).

The graph in figure 5 elaborates that for low load, there is no need for maintaining QoS parameters as all the routing techniques including conventional AODV produces good results. But as the load is increased to $500 \mathrm{kbps}$ which is roughly $2 \%$ of the capacity of the node, conventional AODV's PDR falls bellow 70\%. Although performance of fuzzy and entropy routing is better than that of pure AODV, performance of our proposed technique clearly outperforms the rest.

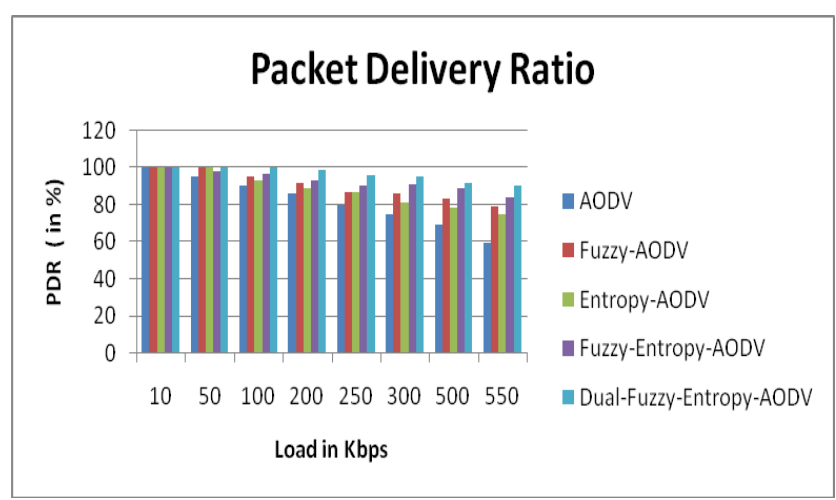

Figure 5: Packet delivery ratio of Proposed Technique Vs Load

It is also clear from the performance graph, that although Fuzzy-Entropy routing truly improves the performance over conventional AODV, Dual-Fuzzy-Entropy-AODV outperforms over the rest .Our algorithm can achieve a packet delivery ratio of $90 \%$ where as AODV's performance goes down to $60 \%$ for a load of 550kbps. Improved results can be attributed to probabilistic and fuzzy based estimation of link quality.

End to End delay observed shows that AODV protocol suffers minimum delay at lower loads as path selection is based on shortest number of hops which is less time consuming .But channel conditions deteriorate as the load increases and hence the quality of the transmission links gets degraded resulting in congestion. AODV's path selection algorithm cannot take into account, congested links resulting out of degraded channel conditions. The advantage of our proposed mechanism is evident as the load increases .

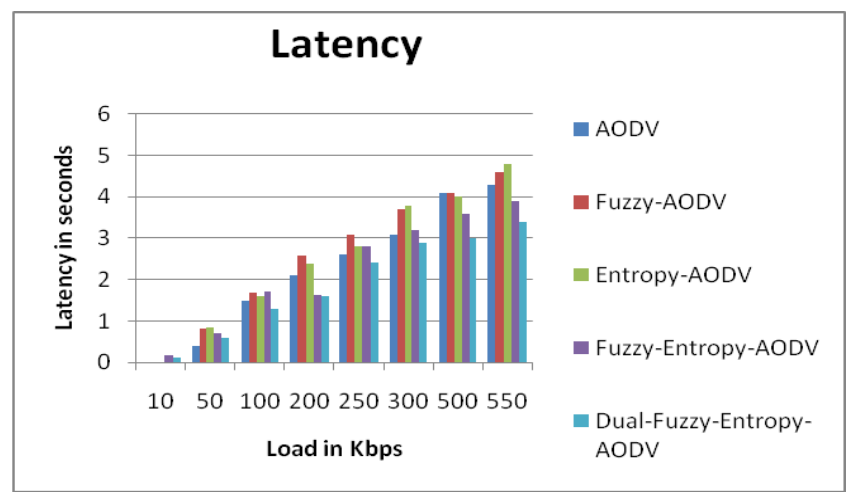

Figure 6: End to End Delay experienced of Proposed Technique Vs Network Load.

To a certain extent Fuzzy-Entropy extension is able to maintain lower latency compared to Fuzzy-AODV and EntropyAODV.But our proposed technique improves the performance over its peers at higher load. AODV suffers the most at increased load conditions.Figure 6 shows the latency incurred.

Figure 7 shows that, AODV again incurs highest control overhead as the network load increases. This increased control overhead is attributed to the fact that AODV will have ,higher number of RERR packets which results in rerouting. This causes more number of control packets occupying the channel than data packets.

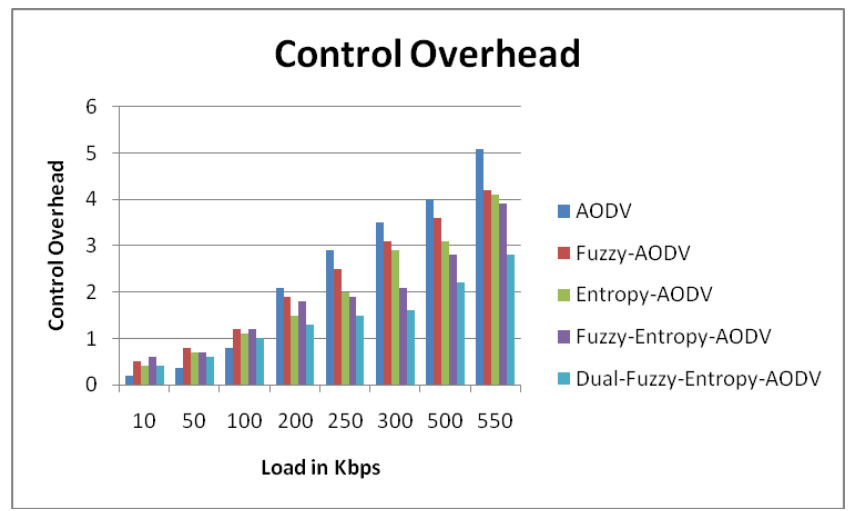

Figure 7: Control Overhead incurred for the proposed Technique Vs Network Load.

Hence AODV experiences highest level of control overhead. As fuzzy based decision making is adopted twice aided with entropy our proposed technique experiences lowest level of control overhead than all its compatriots. Figure 8 shows that how number of sessions affects the performance more than the load. Once number of active connections increases per node, more and more packets are dropped due to interference and queue overflow. This is avoidable if routes with low variations in low interference can be tracked. Therefore the proposed approach performs better than the other techniques even when the number of sessions are high. 


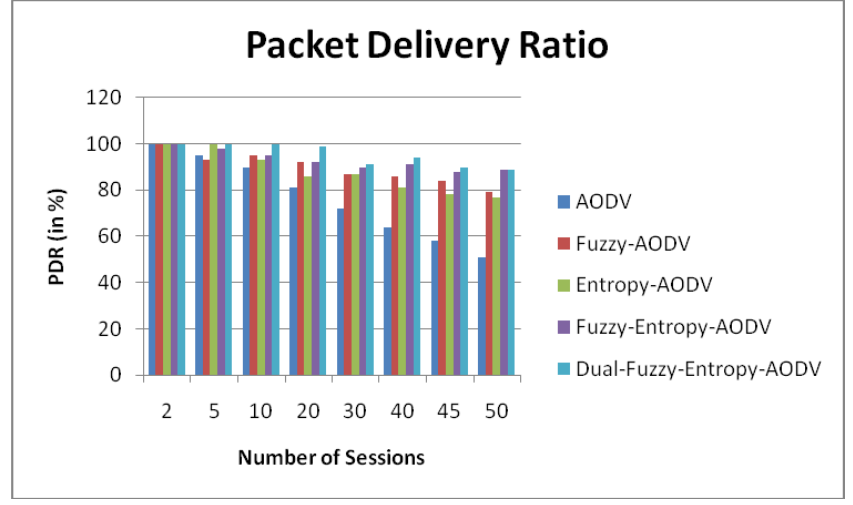

Figure 8: Packet Delivery Ratio of Proposed Technique versus Sessions

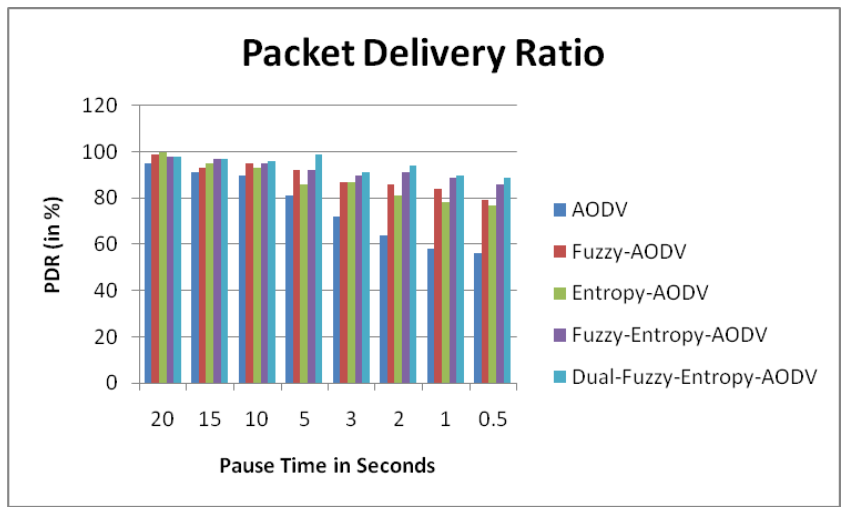

Figure 9: Achieved Packet Delivery Ratio of Proposed Technique versus Pause Time.

Figure 9 shows that high mobility affects the performance of the system and the performance decreases significantly under high mobility. But the proposed system produces better result even under high mobility. Proposed Dual-Fuzzy-Entropy based routing solution is able to perform better than all other routing mechanisms evualated here.

\section{CONCLUSION}

Performance improvement and Quality of Service assurance are two major challenges in MANET protocol design. Several techniques are proposed in this direction and their suitability is proven through experiments and performance evaluation. But there still exists a challenge in this direction as the packet delivery ratio is not been guaranteed close to $100 \%$ under heavy traffic. Fuzzy solutions are an advancement in this direction. Through our experiments we have proved that even fuzzy routing can not assure QoS when more and more nodes demands resources. Combination of Fuzzy and Entropy solves the problem to certain extent and ensure good quality transmission. Our proposed technique on the other hand solves the problem of fuzziness and ever changing state and parameters of the nodes and produces much better results in comparison to the other conventional solutions in this direction. Experiments also reveal that under high topological stability and low load, performance improvement alternatives are not essential but under moderate and heavy traffic, this becomes absolutely mandatory. There may be several other parameters like power, energy, MAC queue size that can directly or indirectly affect the performance. Therefore an optimization technique can be developed to select the optimum parameters before considering them as metric for routing. This work can be a significant direction towards meeting the perfect QoS in MANET under extreme traffic and topology changes.

\section{REFERENCES}

[1] Nicolaos B. Karayiannis, Nagabhushan Kaliyur S.M , " An Entropy-constrined algorithm for routing of communication networks " Computer Communications 29 (2006) 3182-3196.

[2] Cerasoli, C.; Dimarogonas, J. ,The generalization of information entropy to MANET metrics, Military Communications Conference, 2008. MILCOM 2008. IEEE , Publication Year: 2008 , Page(s): 1 - 9.

[3] Chao Gui; Baolin Sun; Chunhua Xia; ,An Entropy-Based Long-Life Multipath Routing Algorithm in MANET, Natural Computation, 2008. ICNC '08. Fourth International Conference on ,Publication Year: 2008 , Page(s): 174 - 178

[4] Sreekanth Nadella, "Power-conserving routing of ad hoc mobile wireless networks based on entropy-constrained algorithm “,Ad Hoc Networks 4 (2006) 24-35, Elsevier

[5] Beongku An, Symeon Pappavassiliou, "An EntropyBased Model for Supporting and Evaluating Stability in Mobile Ad-hoc Wireless Networks," IEEE Communications Letters, vol.6, issue 8, pp.328-330, August 2002.

[6] Cao Trong.H.,Young-Cheol Bang et al " A Multi-Rate Routing Protocol with Connection Entropy for Mobile Ad Hoc Networks “ ,ICUIMC 2010 , Jan 14-15 2010,Suwon, Korea ,ACM.

[7] Hua Chen; Baolin Sun; Yue Zeng; Xianying He; An Entropy-Based Fuzzy Controllers QoS Routing Algorithm in MANET, Hybrid Intelligent Systems, 2009. HIS '09. Ninth International Conference on ,Publication Year: 2009 , Page(s): 235 - 239

[8] Timo, R.; Blackmore, K.; Hanlen, L.; On entropy measures for dynamic network topologies: limits to MANET, Communications Theory Workshop, 2005. Proceedings. 6th Australian ,Publication Year: 2005 , Page(s): 95 - 101.

[9] Gasim Alandjani ,Eric E.Johnson ," Fuzzy Routing in Ad Hoc Networks "IEEE 2003.

[10] Damla Turgut, Kimberly Robinson , Mainak Chatterjee , “ An Entropy-based Clustering Scheme in Mobile AHoc Networks ", Journal of Ubiquitous Computing and Intelligence, Vol 1, pages 101-109 2007.

[11] C. Shannon, “ A mathematical theory of communication", volume 27, pages 623-656, 1948.

[12] G.J.Klir, B.Yuan, “ Fuzzy sets and Fuzzy logic Theory and applications", Prentice Hall, Englewood Cliffs, NJ, USA 1995.

[13] C.Gomathy and S.Shanmugavel, " Fuzzy based Priority Scheduler for mobile ad hoc networks" in Proc. of III Intl conf. on Networking, ICN'04, French Caribbean.

[14] Intersil. HFA3861B: Direct Sequence Spread Spectrum Baseband Processor Data Sheet.

[15] N. Wisitpongphan, G. Ferrari1, S. Panichpapiboon, J.S. Parikh, and O.K. Tonguz, "QoS Provisioning using BERBased Routing in Ad Hoc Wireless Networks",IEEE 2005.

[16] Andrias Vraga, OMNeT++, www.omnetpp.org.

[17] Perkins, C., Belding-Royer, E. and Das, S.,"Ad hoc OnDemand Distance Vector Ad hoc Routing (AODV)". RFC 3561, 2003. 